\title{
Efficacy and safety of ferric derisomaltose (FDI) compared with iron sucrose (IS) in patients with iron deficiency anemia after bariatric surgery
}

\author{
Michael Auerbach ${ }^{1}$ (D) Maureen M. Achebe ${ }^{2} \cdot$ Lars L. Thomsen $^{3} \cdot$ Richard J. Derman $^{4}$
}

Received: 30 August 2021 / Revised: 15 December 2021 / Accepted: 17 December 2021 / Published online: 8 January 2022

(c) The Author(s) 2022

\begin{abstract}
Purpose Iron deficiency is common following bariatric surgery, and treatment with intravenous iron is often required. This post hoc analysis of data from two randomized, open-label, multicenter trials evaluated the efficacy and safety of ferric derisomaltose (FDI; formerly iron isomaltoside 1000) versus iron sucrose (IS) over 4 weeks in adults with iron deficiency anemia (IDA) resulting from prior bariatric surgery.

Materials and methods Data were pooled for participants who received FDI or IS in the PROVIDE or FERWON-IDA trials for the treatment of IDA post bariatric surgery. Efficacy outcomes included changes in hemoglobin (Hb) and iron parameters; safety outcomes included the incidence of adverse drug reactions (ADRs), serious or severe hypersensitivity reactions (HSRs), and hypophosphatemia.

Results The analysis included 159 patients. Mean (standard deviation) cumulative iron doses were $1199( \pm 347) \mathrm{mg}$ for FDI and $937( \pm 209) \mathrm{mg}$ for IS. Compared with IS, FDI resulted in a faster and more pronounced Hb response, and a higher proportion of responders ( $\mathrm{Hb}$ level increase $\geq 2 \mathrm{~g} / \mathrm{dL}$ from baseline) at all time points. The incidence of ADRs was similar with FDI and IS (15.1\% and 18.2\%, respectively), with no serious ADRs or serious or severe HSRs reported. The incidence of hypophosphatemia was low and similar in both treatment groups, with no cases of severe hypophosphatemia observed. Conclusions In patients with IDA resulting from bariatric surgery, FDI produced a faster and more pronounced $\mathrm{Hb}$ response than IS. Both FDI and IS were well tolerated.
\end{abstract}

Keywords Iron deficiency anemia $\cdot$ Ferric derisomaltose $\cdot$ Iron isomaltoside $\cdot$ Iron sucrose $\cdot$ Bariatric surgery

Key points

- This analysis assessed intravenous iron (FDI vs IS) use for IDA post bariatric surgery.

- The hematological response was faster and more pronounced with FDI versus IS.

- FDI and IS were well tolerated, with no serious adverse drug reactions reported.

- No severe hypophosphatemia occurred in a population that is at risk of low phosphate.

Michael Auerbach

mauerbachmd@abhemonc.com

1 Department of Medicine, Georgetown University School of Medicine, Washington, DC, USA

2 Brigham and Women's Hospital, Dana Farber Cancer Institute, Harvard Medical School, Boston, MA, USA

3 Department of Clinical and Non-Clinical Research, Pharmacosmos A/S, Holbæk, Denmark

4 Thomas Jefferson University, Philadelphia, PA, USA

\section{Introduction}

Iron deficiency (ID) is a common cause of anemia following bariatric surgery [1, 2], especially after Roux-en-Y gastric bypass (RYGB), which restricts food intake and nutrient absorption [3-5]. Indeed, Gesquiere et al. reported a 37.2\% incidence of ID within 5 years of RYGB [6].

Multiple factors are associated with the development of ID post bariatric surgery, including a reduction in the surface area available for iron absorption (due to decreased stomach capacity and bypass of the duodenum), an inadequate intake of dietary iron (due to a low tolerance of red meat), and reduced gastric acid secretion [2, 4, 7, 8]. Gastric acid is required for the conjugation of iron to vitamin $\mathrm{C}$, amino acids, and sugar, which prevent its conversion to unabsorbable ferric hydroxide in the proximal duodenum [9]. 
In addition, ID is frequently observed in individuals with obesity $[10,11]$, which may be due to adiposity-related chronic inflammation inhibiting the absorption of iron [12, 13]. Pre-existing ID can be exacerbated following bariatric surgery $[10,11]$. ID can have a considerable impact on an individual's health, particularly when the deficiency results in anemia. Anemia can manifest as fatigue, dizziness, and shortness of breath; in severe cases, anemia can lead to neurological damage and even heart failure [14]. Other symptoms include pagophagia (a pathological craving for ice) [15], which can impair dentition, and restless legs syndrome, which interferes with sleep resulting in fatigue and impaired quality of life $[9,16]$. Therefore, treating ID and any associated anemia is essential.

Clinical practice guidelines recommend that ID following bariatric surgery be treated with oral iron or intravenous (IV) iron $[17,18]$. Collaborative guidelines recommend that high doses of oral iron should be the first-line treatment for ID/ iron deficiency anemia (IDA) with IV iron reserved only for individuals with severe intolerance to oral iron or with treatment-refractory ID/IDA [18]. Increasing evidence suggests that these guidelines need revisiting. Daily, high-dose oral iron increases hepcidin levels, which in turn reduces iron absorption [19]. Indeed, studies have reported increased iron absorption with alternate-day versus once- or twice-daily dosing $[20,21]$, suggesting that daily oral iron is unnecessary and even counterproductive.

European guidelines imply that IV iron supplementation can be administered to correct ID without an initial trial of oral iron [17]. Evidence suggests that IV iron may be preferable to oral iron for the treatment of ID/IDA following bariatric surgery. Clinical studies have shown a decline in the ability of individuals to absorb oral iron in the months following bariatric surgery [2, 6, 22]. Additionally, significant gastrointestinal side effects are often reported with oral iron, which can lead to poor treatment adherence [5, 23]. Compared with oral iron, IV iron treatment has been shown to result in fewer adverse events in patients with ID post bariatric surgery, and in faster normalization of iron parameters and a lower reoccurrence of ID in the 12 months following iron supplementation [24]. Furthermore, the IV route avoids exacerbating the existing gastrointestinal perturbations, which are present in individuals whose gastrointestinal tracts have been rerouted [9].

Newer IV iron formulations are approved for administration in high single doses, which minimize the number of infusions needed for iron repletion and the likelihood of requiring retreatment [25-31]. Consequently, high-dose formulations result in fewer visits than low-dose formulations, thereby reducing costs $[25,27,28]$, while increasing convenience for patients and practitioners.

Despite the advantages, there is a degree of reluctance to use IV iron due to the perception that it can cause severe hypersensitivity reactions (HSRs) [9]. In reality, although infusion reactions can occur with all IV iron products, the majority of reactions are minor and easily managed, and serious or severe HSRs are rare [32-34].

Hypophosphatemia is a concern with some IV iron formulations [35-38]. While clinical sequelae are uncommon, especially after only one or two doses, persistent hypophosphatemia after multiple doses (often needed post bariatric surgery) can lead to short- and long-term clinical consequences such as fatigue, muscle weakness, osteomalacia, bone pain, and fractures [35, 36, 38].

Ferric derisomaltose (FDI) (formerly known as iron isomaltoside 1000) is a high-dose IV iron formulation $[39,40]$, which has shown good efficacy and safety in clinical trials for the treatment of ID/IDA across various specialties, including gastroenterology [41, 42]. Two such clinical trials-PROVIDE and FERWON-IDA-compared the efficacy and safety of FDI with that of iron sucrose (IS; a low-dose IV iron formulation) in patients with IDA of various etiologies, including prior bariatric surgery [43-46]. In the PROVIDE and FERWON-IDA trials, FDI demonstrated a more rapid improvement in hematological parameters than IS, with similar low rates of serious or severe HSRs, serious adverse drug reactions (ADRs), and hypophosphatemia [43, 44]. Although the hematological response in FERWON-IDA was, initially, faster with FDI versus IS, the change in hemoglobin $(\mathrm{Hb})$ at the end of the 8-week follow-up period was similar in both treatment groups [44].

The present post hoc analysis was performed on pooled data from the PROVIDE and FERWON-IDA trials to evaluate the efficacy and safety of FDI compared with IS in patients with IDA resulting from prior bariatric surgery.

\section{Materials and methods}

\section{Trial design}

This was a post hoc analysis of pooled data from two prospective, randomized, open-label, comparative, multicenter trials, which evaluated the efficacy and safety of FDI compared with IS in the treatment of IDA: PROVIDE (NCT02130063) and FERWON-IDA (NCT02940886) [43, 44]. This analysis was conducted in the trial participants with IDA resulting from prior bariatric surgery (gastric bypass, gastric banding, obesity surgery, metabolic surgery, or gastrectomy [sleeve gastrectomy]) (Table 1). The designs of these two trials have been described previously [43, 44]. This pooled analysis was not pre-specified in the protocols of the two trials. 
Table 1 Trials included in the pooled analysis

\begin{tabular}{|c|c|c|}
\hline & PROVIDE & FERWON-IDA \\
\hline Analysis population & $\begin{array}{l}\text { IDA resulting from prior bariatric surgery (gastric bypass, } \\
\text { obesity surgery, or gastrectomy [sleeve gastrectomy]) }\end{array}$ & $\begin{array}{l}\text { IDA resulting from prior bariatric surgery (gastric banding, } \\
\text { gastric bypass, metabolic surgery, or gastrectomy [sleeve } \\
\text { gastrectomy]) }\end{array}$ \\
\hline IDA criteria & $\mathrm{Hb}<11.0 \mathrm{~g} / \mathrm{dL}, \mathrm{TSAT}<20 \%$, and $s$-ferritin $<100 \mathrm{ng} / \mathrm{mL}$ & $\mathrm{Hb} \leq 11.0 \mathrm{~g} / \mathrm{dL}, \mathrm{TSAT}<20 \%$, and $s$-ferritin $<100 \mathrm{ng} / \mathrm{mL}$ \\
\hline \multirow[t]{2}{*}{ Patient numbers } & FDI: $\mathrm{N}=27$ & FDI: $N=66$ \\
\hline & IS: $\mathrm{N}=19$ & IS: $\mathrm{N}=47$ \\
\hline \multirow[t]{2}{*}{ IV iron dosing } & $\begin{array}{l}\text { FDI: cumulative dose of } 1000 \mathrm{mg}, 1500 \mathrm{mg} \text {, } \\
\text { or } 2000 \mathrm{mg} \text { depending on } \mathrm{Hb} \text { level and body weight }\end{array}$ & FDI: single dose of $1000 \mathrm{mg}$ \\
\hline & $\begin{array}{l}\text { IS: cumulative dose according to label and calculated } \\
\text { using the Ganzoni formula (maximum cumulative dose: } \\
2000 \mathrm{mg} \text { ) }\end{array}$ & $\begin{array}{l}\text { IS: cumulative dose according to label (recommended } \\
\text { cumulative dose: } 1000 \mathrm{mg} \text { ) }\end{array}$ \\
\hline Trial duration & 5 weeks & $10-15$ weeks \\
\hline Reference & Derman R, et al. Am J Hematol 2017; 92(3):286-91 & Auerbach M, et al. Am J Hematol 2019; 94(9):1007-14 \\
\hline
\end{tabular}

FDI, ferric derisomaltose/iron isomaltoside 1000; Hb, hemoglobin; IDA, iron deficiency anemia; IS, iron sucrose; IV, intravenous; N, number of patients; $s$-ferritin, serum ferritin; TSAT, transferrin saturation

\section{Participants}

The trials were conducted at 123 sites in the USA; the 74 sites that treated post bariatric surgery patients were included in this pooled analysis. Adults $\geq 18$ years of age with IDA of various etiologies, and with a documented history of intolerance or a lack of response to oral iron, or with a clinical need for rapid repletion of iron stores, were eligible for enrolment. IDA was defined as an $\mathrm{Hb}$ concentration $<11.0 \mathrm{~g} / \mathrm{dL}$ (in PROVIDE) or $\leq 11.0 \mathrm{~g} / \mathrm{dL}$ (in FERWON-IDA), transferrin saturation (TSAT) $<20 \%$, and serum ferritin $(s$-ferritin) $<100 \mathrm{ng} / \mathrm{mL}$ (Table 1$)$. The full list of inclusion and exclusion criteria is presented in the publication for each trial $[43,44]$.

\section{Interventions}

Participants were randomized 2:1 to receive treatment with FDI (Monofer ${ }^{\circledR} /$ Monoferric $^{\circledR}$, Pharmacosmos A/S, Holbæk, Denmark [39, 40]) or IS (Venofer ${ }^{\circledR}$, American Regent, Shirley, New York, USA [45, 46]). In the PROVIDE trial, FDI was administered weekly as $1000 \mathrm{mg}$ IV infusions or $500 \mathrm{mg}$ bolus injections, to achieve a cumulative dose of $1000 \mathrm{mg}, 1500 \mathrm{mg}$, or $2000 \mathrm{mg}$ depending on $\mathrm{Hb}$ concentration and body weight. IS was administered as $200 \mathrm{mg}$ IV infusions up to two times per week to achieve a cumulative dose calculated using the Ganzoni formula; the maximum cumulative dose of IS was $2000 \mathrm{mg}$. In the FERWONIDA trial, FDI was administered as a single $1000 \mathrm{mg} \mathrm{IV}$ infusion at baseline. IS was administered as $200 \mathrm{mg}$ IV injections, which were repeated up to five times. The recommended cumulative dose of IS was $1000 \mathrm{mg}$. During the trials, iron supplementation with products other than the investigational drug was prohibited, as were blood transfusion, and erythropoiesis stimulating agents.

\section{Objective and endpoints}

This pooled analysis evaluated the efficacy and safety of FDI compared with IS in a population with IDA after bariatric surgery. The analysis documented all doses of IV iron administered at baseline and at Weeks 1,2, and 3. All outcomes in the post hoc analysis were assessed at the post-baseline time points that were shared by the two trials (Weeks 1, 2, and 4). Efficacy outcomes included the change in $\mathrm{Hb}, s$-ferritin, and TSAT levels from baseline, the proportion of responders (defined as participants with an $\mathrm{Hb}$ concentration increase $\geq 2 \mathrm{~g} / \mathrm{dL}$ from baseline), the time to achieve a treatment response, and the proportion of participants achieving target iron parameters ( $s$-ferritin $\geq 100 \mathrm{ng} / \mathrm{mL}$ and TSAT of $20-50 \%$ ). Safety outcomes included the incidence of ADRs (i.e., the proportion of patients with ADRs), the incidence of treatment-emergent serious or severe HSRs, and laboratory assessments, such as the change in serum calcium ( $s$-calcium) concentration from baseline, and the incidence of hypophosphatemia (serum phosphate $[s$-phosphate $]<2.0 \mathrm{mg} / \mathrm{dL}$ ) and severe hypophosphatemia ( $s$-phosphate $<1.0 \mathrm{mg} / \mathrm{dL}$ ). Serious or severe HSRs were defined by a standardized set of Medical Dictionary for Regulatory Activities (MedDRA) terms. The MedDRA terms are listed in the supplementary material of the FERWON-IDA trial publication [44]. 


\section{Data analysis sets}

Safety analyses were conducted on the safety analysis set (SAS; $\mathrm{N}=159$ ), defined as all randomized participants who received at least one dose of the trial medication. Efficacy analyses were conducted on the full analysis set (FAS; $\mathrm{N}=159$ ), which included all participants in the SAS who had at least one post-baseline $\mathrm{Hb}$ measurement. In this pooled analysis, the SAS and the FAS represented the same population.

\section{Statistical analyses}

Data are presented as mean (standard deviation [SD]) and least squares mean (95\% confidence interval) for continuous variables, and as the number and percentage of participants for categorical variables.

Baseline laboratory parameters were compared between the treatment groups using a Wilcoxon rank-sum test. A mixed model for repeated measures with trial, treatment, and day as factors, treatment-by-day and baseline value-byday interactions, and baseline value as covariate was used to compare the mean changes in $\mathrm{Hb}, s$-ferritin, TSAT, and $s$-calcium. The proportion of responders, and participants with $s$-ferritin $\geq 100 \mathrm{ng} / \mathrm{mL}$ and TSAT of $20-50 \%$, were compared between the treatment groups using a Fisher's exact test. Time to treatment response was estimated using a Kaplan-Meier method, and the treatment groups were compared using a log-rank test. The incidences of ADRs, serious or severe HSRs, and hypophosphatemia were compared between the treatment groups using a Fisher's exact test. All statistical tests were two-tailed with a significance level of 0.05 . All analyses were performed using SAS software (version 9.4).

\section{Results}

\section{Population}

This pooled analysis included a total of 159 patients with IDA occurring after bariatric surgery: 93 received FDI and 66 IS. Demographics and baseline laboratory parameters are summarized in Table 2. Caucasian women constituted the majority of the population, and the most common type of bariatric surgery was gastric bypass ( $>85 \%$ of cases). On average, bariatric surgery had been conducted $>8.5$ years before the trial. Baseline characteristics were comparable among the treatment groups, although $s$-ferritin and TSAT levels were numerically higher in the FDI group compared with the IS group. $S$-phosphate and $s$-calcium levels were similar in both treatment groups and were within the reference ranges defined by the central laboratories used in the two trials.

\section{Exposure to iron}

The mean (SD) cumulative iron dose was $1199( \pm 347)$ mg with FDI and $937( \pm 209) \mathrm{mg}$ with IS during the first 3 weeks of the trials. Of the participants treated with FDI, $73.1 \%$ (68/93) received a single administration and 26.9\% (25/93) received two administrations. The IS group received between two and seven administrations; the majority of individuals $(68.2 \% ; \mathrm{n}=45 / 66)$ received five administrations.

\section{Change in hemoglobin}

$\mathrm{Hb}$ concentration increased more rapidly and to a greater extent from baseline to Week 4 with FDI compared with IS. The increase in $\mathrm{Hb}$ was significantly higher with FDI than IS at Weeks $1(\mathrm{p}<0.01), 2(\mathrm{p}<0.0001)$, and $4(\mathrm{p}<0.01$; Fig. 1). The proportion of responders (defined as participants with an $\mathrm{Hb}$ concentration increase $\geq 2 \mathrm{~g} / \mathrm{dL}$ from baseline) was higher with FDI than with IS at Weeks 1, 2, and 4. The difference was statistically significant at Week 2 ( $p<0.0001$; Table 3 ). The time to $\mathrm{Hb}$ response was significantly shorter with FDI versus IS $(p<0.01)$. The number of participants responding at Weeks 1, 2, and 4 was 5, 29, and 30 with FDI, and 0,4 , and 33 with IS.

\section{Changes in serum ferritin and transferrin saturation}

$S$-ferritin concentration and TSAT increased more rapidly and to a greater extent with FDI versus IS at Weeks 1 and 2 ( $s$-ferritin, $\mathrm{p}<0.0001$ at both time points; TSAT, $\mathrm{p}<0.0001$ at Week 1 , and $\mathrm{p}<0.01$ at Week 2), but there were no significant differences between the groups at Week 4 (Fig. 1). At all time points assessed, the proportion of participants achieving target iron parameters ( $s$-ferritin $\geq 100 \mathrm{ng} / \mathrm{mL}$ and TSAT of 20-50\%) was higher in the FDI group, and the difference was statistically significant at Weeks 1 and 2 $(\mathrm{p}<0.0001$ at both time points; Table 3$)$.

\section{Adverse drug reactions and hypophosphatemia}

The incidence of ADRs was similar, $<20 \%$ in the FDI and IS groups, although the number of ADRs was twice as high with IS compared with FDI (Table 4). None of the ADRs were considered serious, and no serious or severe HSRs were reported. The most common ADRs ( $\geq 3 \%$ in any group) included constipation, myalgia, nausea, headache, dysgeusia, fatigue, hyperhidrosis, and vomiting, with no significant differences observed between the FDI and IS treatment groups (Table 4). 
Table 2 Demographics and baseline laboratory parameters

\begin{tabular}{|c|c|c|}
\hline & $\begin{array}{l}\text { FDI } \\
(\mathrm{N}=93)\end{array}$ & $\begin{array}{l}\text { IS } \\
(\mathrm{N}=66)\end{array}$ \\
\hline \multicolumn{3}{|l|}{ Demographics } \\
\hline Age (years) & $47.6(10.8)$ & $45.3(10.2)$ \\
\hline \multicolumn{3}{|l|}{ Gender, N (\%) } \\
\hline Women & $91(97.8)$ & $64(97.0)$ \\
\hline Men & $2(2.2)$ & $2(3.0)$ \\
\hline \multicolumn{3}{|l|}{ Race, N (\%) } \\
\hline White & $78(83.9)$ & $51(77.3)$ \\
\hline Black or African American & $13(14.0)$ & $14(21.2)$ \\
\hline Native Hawaiian or other Pacific Islander & $0(0.0)$ & $1(1.5)$ \\
\hline Other & $2(2.2)$ & $0(0.0)$ \\
\hline Weight (kg) & $89.9(22.6)$ & $89.5(22.3)$ \\
\hline BMI $\left(\mathrm{kg} / \mathrm{m}^{2}\right)$ & $33.5(8.0)$ & $33.3(8.5)$ \\
\hline \multicolumn{3}{|l|}{ Bariatric surgery, N (\%) } \\
\hline Gastric bypass & $80(86.0)$ & $57(86.4)$ \\
\hline Gastric banding & $3(3.2)$ & $2(3.0)$ \\
\hline Obesity surgery & $3(3.2)$ & $0(0.0)$ \\
\hline Metabolic surgery & $3(3.2)$ & $4(6.1)$ \\
\hline Gastrectomy (sleeve gastrectomy) & $4(4.3)$ & $3(4.5)$ \\
\hline Time since bariatric surgery (years) ${ }^{\mathrm{a}}$ & $9.7(6.7)(n=92)$ & $8.6(7.3)(n=66)$ \\
\hline \multicolumn{3}{|l|}{ Laboratory parameters } \\
\hline $\mathrm{Hb}(\mathrm{g} / \mathrm{dL})$ & $9.3(1.0)$ & $9.2(1.3)$ \\
\hline$S$-ferritin $(\mathrm{ng} / \mathrm{mL})$ & $9.3(14.3)$ & $6.2(4.0)$ \\
\hline TSAT $(\%)$ & $6.9(13.2)$ & $5.1(2.8)$ \\
\hline$S$-phosphate (mg/dL) & $3.7(0.5)(\mathrm{n}=89)$ & $3.6(0.6)(n=63)$ \\
\hline$S$-calcium (mg/dL) & $8.9(0.4)(n=89)$ & $8.9(0.4)(n=63)$ \\
\hline
\end{tabular}

Data are presented for the FAS; data presented are mean (SD) unless otherwise stated

${ }^{a}$ Time between the date of surgery (all types of bariatric surgery) and the date of first dose in the trial BMI, body mass index; FAS, full analysis set; FDI, ferric derisomaltose/iron isomaltoside 1000; Hb, hemoglobin; IS, iron sucrose; N, number of patients; SD, standard deviation; $s$-calcium, serum calcium; $s$-ferritin, serum ferritin; $s$-phosphate, serum phosphate; TSAT, transferrin saturation
The incidence of hypophosphatemia ( $s$-phosphate $<2.0 \mathrm{mg} / \mathrm{dL})$ was $0.0 \%(0 / 91)$ in the FDI group and $1.6 \%(1 / 63)$ in the IS group at Week 1, and 3.3\% $(3 / 91)$ in the FDI group and $0.0 \%(0 / 59)$ in the IS group at Week 2. At both time points, the differences between the treatment groups were not statistically significant. At Week 4, there were no cases of hypophosphatemia. None of the participants developed severe hypophosphatemia $(s$-phosphate $<1.0 \mathrm{mg} / \mathrm{dL})$.

\section{Serum calcium}

$S$-calcium was stable across the 4 weeks and remained within reference range in both treatment groups (mean levels remained at approximately $9 \mathrm{mg} / \mathrm{dL}$ with FDI and IS); no significant differences were observed between the groups.

\section{Discussion}

In this post hoc analysis of pooled data from the PROVIDE and FERWON-IDA trials, FDI resulted in faster and more pronounced hematological responses compared with IS in the subgroup with IDA following prior bariatric surgery. The time to achieve an $\mathrm{Hb}$ increase $\geq 2 \mathrm{~g} / \mathrm{dL}$ was also significantly shorter with FDI than with IS. These data are consistent with the main analyses of the PROVIDE and FERWON-IDA trials, which included patients with IDA of various etiologies [43, 44].

The opportunity to give higher doses of FDI in fewer administrations, within a shorter time period, compared with IS, possibly accounted for the faster and more pronounced improvements in hematological parameters observed with FDI. At least five infusions of IS would be required to achieve the same dose as a single $1000 \mathrm{mg}$ 

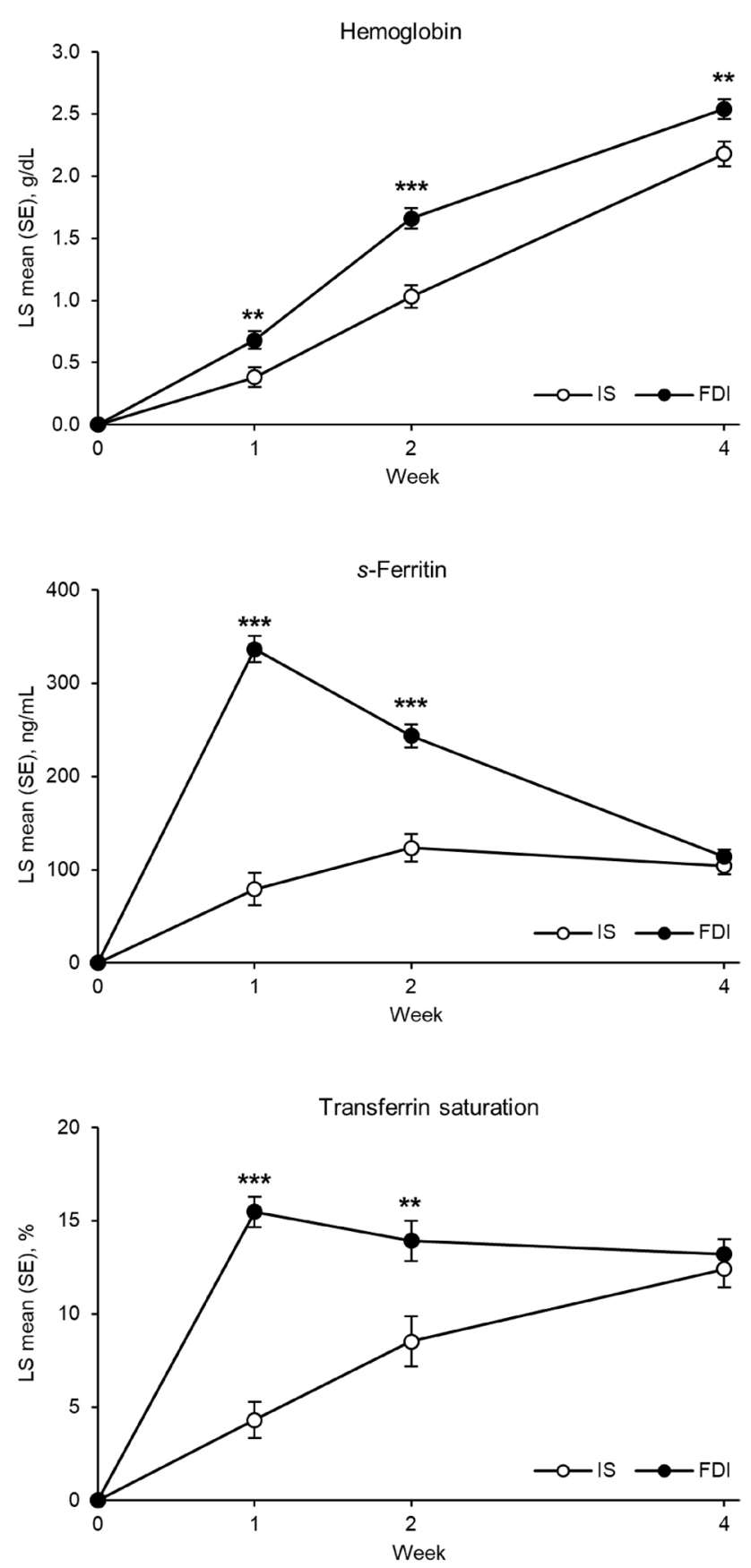

Fig. 1 LS mean change in hematological parameters from baseline over 4 weeks. ${ }^{* *} \mathrm{p}<0.01, * * * \mathrm{p}<0.001$ versus IS; estimates from mixed model for repeated measures with study, treatment and day as factors, treatment*day and baseline*day interactions, and baseline value as covariate. Data are presented for the FAS. FAS, full analysis set; FDI, ferric derisomaltose; IS, iron sucrose; LS, least squares; SE, standard error

infusion of FDI. In this analysis, the modal number of infusions was one with FDI and five with IS. Reduced dosing frequency is a key advantage of FDI over IS, which in turn can reduce costs [27, 28].
Table 3 Frequency of responders and participants achieving target iron parameters

\begin{tabular}{|c|c|c|c|}
\hline & $\begin{array}{l}\text { FDI } \\
\text { n/N (\%) }\end{array}$ & $\begin{array}{l}\text { IS } \\
\text { n/N (\%) }\end{array}$ & P-value ${ }^{a}$ \\
\hline \multicolumn{4}{|c|}{ Participants with $\mathrm{Hb}$ level increase $\geq 2 \mathrm{~g} / \mathrm{dL}$ from baseline } \\
\hline Week 1 & $5 / 91(5.5)$ & $0 / 62(0.0)$ & 0.0810 \\
\hline Week 2 & 33/91 (36.3) & $4 / 61(6.6)$ & $<0.0001$ \\
\hline Week 4 & $63 / 91(69.2)$ & $37 / 61(60.7)$ & 0.2989 \\
\hline \multicolumn{4}{|c|}{ Participants with $s$-ferritin $\geq 100 \mathrm{ng} / \mathrm{mL}$ and TSAT of $20-50 \%$} \\
\hline Week 1 & $56 / 88(63.6)$ & $3 / 63(4.8)$ & $<0.0001$ \\
\hline Week 2 & $42 / 91(46.2)$ & $5 / 59(8.5)$ & $<0.0001$ \\
\hline Week 4 & 26/90 (28.9) & $14 / 60(23.3)$ & 0.5722 \\
\hline
\end{tabular}

Data are presented for the FAS

${ }^{a}$ FDI versus IS using a Fisher's exact test

FAS, full analysis set; FDI, ferric derisomaltose/iron isomaltoside 1000; Hb, hemoglobin; IS, iron sucrose; n, number of responders; N, number of patients; $s$-ferritin, serum ferritin; TSAT, transferrin saturation

Table 4 Incidence of ADRs over 4 weeks from first exposure

\begin{tabular}{|c|c|c|c|c|c|}
\hline & \multicolumn{2}{|l|}{$\begin{array}{l}\text { FDI } \\
(\mathrm{N}=93)\end{array}$} & \multicolumn{2}{|l|}{$\begin{array}{l}\text { IS } \\
(\mathrm{N}=66)\end{array}$} & \multirow[t]{2}{*}{ P-value } \\
\hline & $\mathrm{N}(\%)$ & $\mathrm{E}$ & $\mathrm{N}(\%)$ & $\mathrm{E}$ & \\
\hline ADRs & $14(15.1)$ & 20 & $12(18.2)$ & 43 & 0.6657 \\
\hline Serious ADRs & $0(0.0)$ & 0 & $0(0.0)$ & 0 & NA \\
\hline \multicolumn{6}{|c|}{ ADRs (MedDRA preferred term) with incidence $\geq 3 \%$ in any group } \\
\hline Constipation & $4(4.3)$ & 4 & $0(0.0)$ & 0 & 0.1420 \\
\hline Myalgia & $1(1.1)$ & 1 & $2(3.0)$ & 3 & 0.5705 \\
\hline Nausea & $1(1.1)$ & 1 & $3(4.5)$ & 4 & 0.3080 \\
\hline Headache & $1(1.1)$ & 1 & $2(3.0)$ & 2 & 0.5705 \\
\hline Dysgeusia & $0(0.0)$ & 0 & $2(3.0)$ & 4 & 0.1708 \\
\hline Fatigue & $0(0.0)$ & 0 & $2(3.0)$ & 4 & 0.1708 \\
\hline Hyperhidrosis & $0(0.0)$ & 0 & $2(3.0)$ & 2 & 0.1708 \\
\hline Vomiting & $0(0.0)$ & 0 & $2(3.0)$ & 2 & 0.1708 \\
\hline
\end{tabular}

Data are presented for the SAS

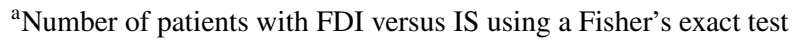
ADR, adverse drug reaction; E, number of events, FDI, ferric derisomaltose/iron isomaltoside 1000; IS, iron sucrose; MedDRA, Medical Dictionary for Regulatory Activities; N, number of patients; NA, not applicable; SAS, safety analysis set

Another high-dose IV iron product-ferric carboxymaltose (FCM) - has demonstrated effectiveness in bariatric surgery patients with ID, with or without anemia [24, 47]. Although FDI and FCM have not been compared directly in a bariatric surgery population, clinical trials across various therapeutic indications have shown that FDI and FCM have similar efficacy in the treatment of IDA of various etiologies $[37,48,49]$. 
The population in this analysis reflects the expected characteristics of patients with IDA resulting from bariatric surgery. The majority of patients were women, who are at higher risk of developing IDA than men [1, 6]. In this analysis, $>85 \%$ had undergone a gastric bypass procedure-the operation associated with the highest incidence of IDA [5, $6,10]$.

In this pooled analysis, FDI and IS were well tolerated with a similar incidence of ADRs (15.1\% and $18.2 \%$, respectively), and no serious ADRs were observed. Similar ADR profiles were also reported in the mixed IDA populations in the PROVIDE and FERWON-IDA parent trials [43, 44].

The potential for serious or severe HSRs with IV iron is a concern among medical professionals though, in reality, serious or severe HSRs are rare [32, 50]. In this pooled analysis, no serious or severe HSRs were reported with either formulation. These data are consistent with the current findings in populations with IDA of various etiologies. An analysis of data from high-quality randomized controlled trials found a low incidence of serious or moderate-to-severe HSRs (0.2-1.7\%) with newer IV iron formulations, and no statistically significant differences between formulations [32]. Furthermore, a comprehensive meta-analysis of data from trials enrolling more than 8500 patients with IDA of various etiologies confirmed the low incidence of serious or severe HSRs (0.6-1.6\%) with modern IV iron formulations [50]. Reports of IV iron-induced HSRs specifically in the bariatric surgery population also show low rates of serious or severe HSRs [24, 47], with no differences from the mixed IDA population.

Hypophosphatemia is a concern with certain IV iron products and can have important clinical consequences, particularly when the hypophosphatemia is severe and persistent [38]. Bariatric surgery patients may be particularly susceptible to hypophosphatemia in the context of IV iron treatment, as they often develop secondary hyperparathyroidism due to vitamin D deficiency and calcium malabsorption, which can lower $s$-phosphate levels $[51,52]$. In this analysis of post bariatric surgery patients, rates of hypophosphatemia were low with FDI and IS, and were consistent with the rates observed in the mixed IDA populations in the PROVIDE and FERWON-IDA trials [43, 44]. Importantly, no cases of severe hypophosphatemia were observed.

The present analysis has limitations. The analysis was conducted post hoc, which precludes robust conclusions. However, the results are consistent with the preponderance of published evidence demonstrating the efficacy of IV iron in the treatment of ID/IDA following bariatric surgery [24, 47].

Another limitation is the short treatment period used in this analysis. Bariatric surgery patients are highly susceptible to ID and, therefore, may require re-treatment with IV iron. Consequently, it is important to understand the long-term efficacy and safety of IV iron in this population. Although this analysis did not explore the long-term effects of FDI and IS, a 6-month extension study (FERWON-EXT) found that re-dosing patients with FDI resulted in rapid improvements in $\mathrm{Hb}$ levels and low rates of ADRs [53]. FERWON-EXT enrolled patients from the PROVIDE, FERWON-IDA, and FERWON-NEPHRO trials [53], and included individuals with IDA resulting from prior bariatric surgery.

In conclusion, in patients with IDA following bariatric surgery, FDI treatment was delivered in fewer visits and was associated with a faster and more pronounced hematological response than IS. FDI was well tolerated with a similar incidence of ADRs to IS and no cases of severe hypophosphatemia, or serious or severe HSRs.

Acknowledgements The authors would like to thank Jens-Kristian Slott Jensen, Slott Stat, Denmark for statistical support, and 'Cambridge - a Prime Global Agency', Cambridge, UK, for medical writing assistance.

Author contributions M.A., M.M.A., and R.J.D. were investigators in the PROVIDE and FERWON-IDA trials. For the post hoc analysis, they contributed to the interpretation of data, revision of the manuscript for important intellectual content, approval of the final version for submission, and agreed to be accountable for all aspects of the work. L.L.T. contributed to the conception, design, conduct, and reporting of the PROVIDE and FERWON-IDA trials. For the post hoc analysis, he contributed to the interpretation of data, revision of the manuscript for important intellectual content, approval of the final version for submission, and agreed to be accountable for all aspects of the work.

Funding This work was funded by Pharmacosmos A/S.

\section{Declarations}

Conflicts of interest M.A. receives research funding for data management from Covis Pharma and has given educational non-promotional talks for Pharmacosmos A/S and Pfizer. M.M.A. has been on scientific advisory boards for Pharmacosmos A/S. L.L.T. is employed by Pharmacosmos A/S. R.J.D. has no conflicts of interest to declare.

Ethical approval All procedures performed in studies involving human participants were in accordance with the ethical standards of the institutional and/or national research committee and with the 1964 Helsinki declaration and its later amendments or comparable ethical standards.

Informed consent Informed consent was obtained from all individual participants included in the studies.

Open Access This article is licensed under a Creative Commons Attribution 4.0 International License, which permits use, sharing, adaptation, distribution and reproduction in any medium or format, as long as you give appropriate credit to the original author(s) and the source, provide a link to the Creative Commons licence, and indicate if changes were made. The images or other third party material in this article are included in the article's Creative Commons licence, unless indicated otherwise in a credit line to the material. If material is not included in the article's Creative Commons licence and your intended use is not permitted by statutory regulation or exceeds the permitted use, you will 
need to obtain permission directly from the copyright holder. To view a copy of this licence, visit http://creativecommons.org/licenses/by/4.0/.

\section{References}

1. Avgerinos DV, Llaguna OH, Seigerman M, et al. Incidence and risk factors for the development of anemia following gastric bypass surgery. World J Gastroenterol. 2010;16(15):1867-70.

2. Ruz M, Carrasco F, Rojas P, et al. Iron absorption and iron status are reduced after Roux-en-Y gastric bypass. Am J Clin Nutr. 2009;90(3):527-32.

3. Bal BS, Finelli FC, Shope TR, et al. Nutritional deficiencies after bariatric surgery. Nat Rev Endocrinol. 2012;8(9):544-56.

4. Love AL, Billett HH. Obesity, bariatric surgery, and iron deficiency: true, true, true and related. Am J Hematol. 2008;83(5):403-9.

5. Stein J, Stier C, Raab H, et al. Review article: the nutritional and pharmacological consequences of obesity surgery. Aliment Pharmacol Ther. 2014;40(6):582-609.

6. Gesquiere I, Lannoo M, Augustijns P, et al. Iron deficiency after Roux-en-Y gastric bypass: insufficient iron absorption from oral iron supplements. Obes Surg. 2014;24(1):56-61.

7. Behrns KE, Smith CD, Sarr MG. Prospective evaluation of gastric acid secretion and cobalamin absorption following gastric bypass for clinically severe obesity. Dig Dis Sci. 1994;39(2):315-20.

8. Ortega J, Ortega-Evangelio G, Cassinello N, et al. What are obese patients able to eat after Roux-en-Y gastric bypass? Obes Facts. 2012;5(3):339-48.

9. Auerbach M, Deloughery T. Single-dose intravenous iron for iron deficiency: a new paradigm. Hematology Am Soc Hematol Educ Program. 2016;2016(1):57-66.

10. Enani G, Bilgic E, Lebedeva E, et al. The incidence of iron deficiency anemia post-Roux-en-Y gastric bypass and sleeve gastrectomy: a systematic review. Surg Endosc. 2020;34(7):3002-10.

11. Gowanlock Z, Lezhanska A, Conroy M, et al. Iron deficiency following bariatric surgery: a retrospective cohort study. Blood Adv. 2020;4(15):3639-47.

12. McClung JP, Karl JP. Iron deficiency and obesity: the contribution of inflammation and diminished iron absorption. Nutr Rev. 2009;67(2):100-4.

13. Zimmermann MB, Zeder C, Muthayya S, et al. Adiposity in women and children from transition countries predicts decreased iron absorption, iron deficiency and a reduced response to iron fortification. Int J Obes (Lond). 2008;32(7):1098-104.

14. National Heart, Lung, and Blood Institute (NHLBI). Your guide to anemia: National Institutes of Health $(\mathrm{NIH})$. NIH publication number: 11-7629; 2011.

15. Rabel A, Leitman SF, Miller JL. Ask about ice, then consider iron. J Am Assoc Nurse Pract. 2016;28(2):116-20.

16. Allen RP, Walters AS, Montplaisir J, et al. Restless legs syndrome prevalence and impact: REST general population study. Arch Intern Med. 2005;165(11):1286-92.

17. Fried M, Yumuk V, Oppert JM, et al. European Association for the Study of Obesity; International Federation for the Surgery of Obesity - European Chapter. Interdisciplinary European Guidelines on metabolic and bariatric surgery. Obes Facts. 2013;6(5):449-68.

18. Mechanick JI, Apovian C, Brethauer S, et al. Clinical practice guidelines for the perioperative nutrition, metabolic, and nonsurgical support of patients undergoing bariatric procedures - 2019 update: cosponsored by American Association of Clinical Endocrinologists/American College of Endocrinology, The Obesity Society, American Society for Metabolic \& Bariatric
Surgery, Obesity Medicine Association, and American Society of Anesthesiologists - executive summary. Endocr Pract. 2019;25(12):1346-59.

19. Moretti D, Goede JS, Zeder C, et al. Oral iron supplements increase hepcidin and decrease iron absorption from daily or twice-daily doses in iron-depleted young women. Blood. 2015;126(17):1981-9.

20. Stoffel NU, Cercamondi CI, Brittenham G, et al. Iron absorption from oral iron supplements given on consecutive versus alternate days and as single morning doses versus twice-daily split dosing in iron-depleted women: two open-label, randomised controlled trials. Lancet Haematol. 2017;4(11):e524-33.

21. Uyoga MA, Mikulic N, Paganini D, et al. The effect of iron dosing schedules on plasma hepcidin and iron absorption in Kenyan infants. Am J Clin Nutr. 2020;112(4):1132-41.

22. Ruz M, Carrasco F, Rojas P, et al. Heme- and nonheme-iron absorption and iron status 12 mo after sleeve gastrectomy and Roux-en-Y gastric bypass in morbidly obese women. Am J Clin Nutr. 2012;96(4):810-7.

23. Tolkien Z, Stecher L, Mander AP, et al. Ferrous sulfate supplementation causes significant gastrointestinal side-effects in adults: a systematic review and meta-analysis. PLoS One. 2015;10(2): e0117383.

24. Schijns W, Boerboom A, de Bruyn Kops M, et al. A randomized controlled trial comparing oral and intravenous iron supplementation after Roux-en-Y gastric bypass surgery. Clin Nutr. 2020;39(12):3779-85.

25. Bhandari S. Update of a comparative analysis of cost minimization following the introduction of newly available intravenous iron therapies in hospital practice. Ther Clin Risk Manag. 2011;7:501-9.

26. Frigstad SO, Haaber A, Bajor A, et al. The NIMO Scandinavian Study: a prospective observational study of iron isomaltoside treatment in patients with iron deficiency. Gastroenterol Res Pract. 2017;2017:4585164.

27. Pollock RF, Muduma G. A budget impact analysis of parenteral iron treatments for iron deficiency anemia in the UK: reduced resource utilization with iron isomaltoside 1000. Clinicoecon Outcomes Res. 2017;9:475-83.

28. Pollock RF, Muduma G. Intravenous iron treatments for iron deficiency anemia in inflammatory bowel disease: a budget impact analysis of iron isomaltoside 1000 (Monofer) in the UK. Expert Opin Drug Deliv. 2017;14(12):1439-46.

29. Pollock RF, Muduma G. An economic evaluation of iron isomaltoside 1000 versus ferric carboxymaltose in patients with inflammatory bowel disease and iron deficiency anemia in Denmark. Adv Ther. 2018;35(12):2128-37.

30. Pollock RF, Muduma G. A patient-level cost-effectiveness analysis of iron isomaltoside versus ferric carboxymaltose for the treatment of iron deficiency anemia in the United Kingdom. J Med Econ. 2020;23(7):751-9.

31. Pollock RF, Muduma G. An economic analysis of ferric derisomaltose versus ferric carboxymaltose in the treatment of iron deficiency anemia in patients with inflammatory bowel disease in Norway, Sweden, and Finland. Clinicoecon Outcomes Res. 2021;13:9-18.

32. Achebe M, DeLoughery TG. Clinical data for intravenous iron - debunking the hype around hypersensitivity. Transfusion. 2020;60(6):1154-9.

33. Rampton D, Folkersen J, Fishbane S, et al. Hypersensitivity reactions to intravenous iron: guidance for risk minimization and management. Haematologica. 2014;99(11):1671-6.

34. Avni T, Bieber A, Grossman A, et al. The safety of intravenous iron preparations: systematic review and meta-analysis. Mayo Clin Proc. 2015;90(1):12-23. 
35. Glaspy JA, Lim-Watson MZ, Libre MA, et al. Hypophosphatemia associated with intravenous iron therapies for iron deficiency anemia: a systematic literature review. Ther Clin Risk Manag. 2020;16:245-59.

36. Schaefer B, Tobiasch M, Viveiros A, et al. Hypophosphataemia after treatment of iron deficiency with intravenous ferric carboxymaltose or iron isomaltoside - a systematic review and metaanalysis. Br J Clin Pharmacol. 2021;87(5):2256-73.

37. Wolf M, Rubin J, Achebe M, et al. Effects of iron isomaltoside vs ferric carboxymaltose on hypophosphatemia in irondeficiency anemia: two randomized clinical trials. JAMA. 2020;323(5):432-43.

38. Zoller H, Schaefer B, Glodny B. Iron-induced hypophosphatemia: an emerging complication. Curr Opin Nephrol Hypertens. 2017;26(4):266-75.

39. Monofer ${ }^{\circledR}$ (ferric derisomaltose). Summary of Product Characteristics. Pharmacosmos UK Ltd.; 2020.

40. Monoferric ${ }^{\circledR}$ (ferric derisomaltose) injection for intravenous use. Prescribing information. Pharmacosmos A/S; 2020.

41. Auerbach M, Henry D, DeLoughery TG. Intravenous ferric derisomaltose for the treatment of iron deficiency anemia. Am J Hematol. 2021;96(6):727-34.

42. Kassianides X, Bodington R, Bhandari S. An evaluation of ferric derisomaltose as a treatment for anemia. Expert Rev Hematol. 2021;14(1):7-29.

43. Derman R, Roman E, Modiano MR, et al. A randomized trial of iron isomaltoside versus iron sucrose in patients with iron deficiency anemia. Am J Hematol. 2017;92(3):286-91.

44. Auerbach M, Henry D, Derman RJ, et al. A prospective, multicenter, randomized comparison of iron isomaltoside 1000 versus iron sucrose in patients with iron deficiency anemia; the FERWON-IDA trial. Am J Hematol. 2019;94(9):1007-14.

45. Venofer $^{\circledR}$ (iron sucrose) $20 \mathrm{mg}$ iron $/ \mathrm{ml}$, solution for injection or concentrate for solution for infusion. Summary of Product Characteristics. Vifor Pharma UK Ltd.; 2020.
46. Venofer ${ }^{\circledR}$ (iron sucrose) injection, for intravenous use. Prescribing information. American Regent, Inc.; 2019.

47. Malone M, Barish C, He A, et al. Comparative review of the safety and efficacy of ferric carboxymaltose versus standard medical care for the treatment of iron deficiency anemia in bariatric and gastric surgery patients. Obes Surg. 2013;23(9):1413-20.

48. Detlie TE, Lindstrøm JC, Jahnsen ME, et al. Incidence of hypophosphatemia in patients with inflammatory bowel disease treated with ferric carboxymaltose or iron isomaltoside. Aliment Pharmacol Ther. 2019;50(4):397-406.

49. Pollock RF, Muduma G. A systematic literature review and indirect comparison of iron isomaltoside and ferric carboxymaltose in iron deficiency anemia after failure or intolerance of oral iron treatment. Expert Rev Hematol. 2019;12(2):129-36.

50. Pollock RF, Biggar P. Indirect methods of comparison of the safety of ferric derisomaltose, iron sucrose and ferric carboxymaltose in the treatment of iron deficiency anemia. Expert Rev Hematol. 2020;13(2):187-95.

51. Hewitt S, Aasheim ET, Søvik TT, et al. Relationships of serum 25-hydroxyvitamin $\mathrm{D}$, ionized calcium and parathyroid hormone after obesity surgery. Clin Endocrinol (Oxf). 2018;88(3):372-9.

52. Wei JH, Lee WJ, Chong K, et al. High incidence of secondary hyperparathyroidism in bariatric patients: comparing different procedures. Obes Surg. 2018;28(3):798-804.

53. Achebe MM, Glaspy J, Kalra PA, et al. A 6 month extension trial evaluating safety and efficacy of ferric derisomaltose in patients with iron deficiency anemia: the FERWON-EXT trial. Am J Hematol. 2020;95(10):E276-9.

Publisher's note Springer Nature remains neutral with regard to jurisdictional claims in published maps and institutional affiliations. 